\title{
Granular Phase Transition as a Precondition for Segregation
}

\author{
S. Aumaître \\ Laboratoire de Physique Statistique, École Normale Supérieure, F-75231 Paris, France
}

T. Schnautz, C. A. Kruelle, and I. Rehberg

Experimentalphysik V, Universität Bayreuth, D-95440 Bayreuth, Germany

(Received 11 July 2002; revised manuscript received 3 February 2003; published 20 March 2003)

\begin{abstract}
Experimental results are presented on the segregation of a mixture of spheres with two different sizes, rolling on a circularly vibrating table. Beyond a critical density of particles a demixing occurs leading to a clustering of the larger ones. A monodisperse layer of spheres shows a liquid-solid-like phase transition at a slightly lower critical density. These critical particle densities are both found to be independent of the driving frequency, but decrease with increasing vibration amplitude.
\end{abstract}

PACS numbers: $45.70 . \mathrm{Mg}, 05.20 .-\mathrm{y}, 64.75 .+\mathrm{g}$

An accumulation of macroscopic grains, set in motion by an external driving force, can show a surprising behavior. A peculiar phenomenon - called segregation occurs as soon as heterogeneous particles are implied, in geophysical rock avalanches as well as in processed powders in the food or chemical industry [1]. This demixing of grains, which differ in size, density, or surface properties, has been intensively studied since the 1990s in laboratory experiments, e.g., in rotating drums [2] and under vertical [3] or, more recently, horizontal linear [4] excitation. Another phenomenon frequently encountered when handling granular material is the transition from a disordered phase to a more organized state, when the density of grains is increased beyond a critical value. This can be observed, for instance, in monodisperse particles under vertical vibration without gravity [5] or under horizontal translational excitation [6].

Here we report on a model system, consisting of two species of glass beads rolling in a horizontal shaker, where both phenomena, segregation and phase transition, are found to be closely related since they occur at about the same granular density.

A sketch of our experimental device is shown in Fig. 1. The particles in the dish are excited in a horizontal circular vibration, i.e., a circular movement of the entire platform due to a superposition of two sinusoidal vibrations in perpendicular directions. The frequency $\nu$ of the table motion can be tuned from 0.5 to $2 \mathrm{~Hz}$ with a fixed amplitude $A=0.64,0.95,1.27,1.59$, or $1.91 \mathrm{~cm}$. The binary mixture is composed of 19 large black glass spheres of diameter $D=1 \mathrm{~cm}$ and a variable number, $N$, of small transparent glass spheres with diameter $d=0.4 \mathrm{~cm}$. To obtain a size-independent control parameter the total filling fraction $\mu_{\text {tot }}$ is defined as the total cross-sectional area of all small and large spheres divided by the surface of the cell. Initially the large spheres are placed on a regular triangular lattice with $3 \mathrm{~cm}$ spacing between the center of two nearest particles [see inset of Fig. 2(b)]. During the motion, the positions of the large particles are captured with a charge-coupleddevice camera fixed to the moving table. The distance $L_{\mathrm{nn}}(t)$ between nearest neighbors, averaged over all the 19 large spheres, is measured in real time to characterize the segregation [7].

The results of these measurements are shown in Fig. 2 for two different numbers $N$ of small spheres. At low density, $\mu_{\text {tot }}=0.30$, Fig. 2(a) shows large random fluctuations around a value $\bar{L}_{\mathrm{nn}} \approx 2.3 \mathrm{~cm}$, although the particles are placed initially on a $3 \mathrm{~cm}$ lattice. This distance can be considered as a mean free path between large particles. No signs of decay of $L_{\mathrm{nn}}(t)$, as would be expected in a

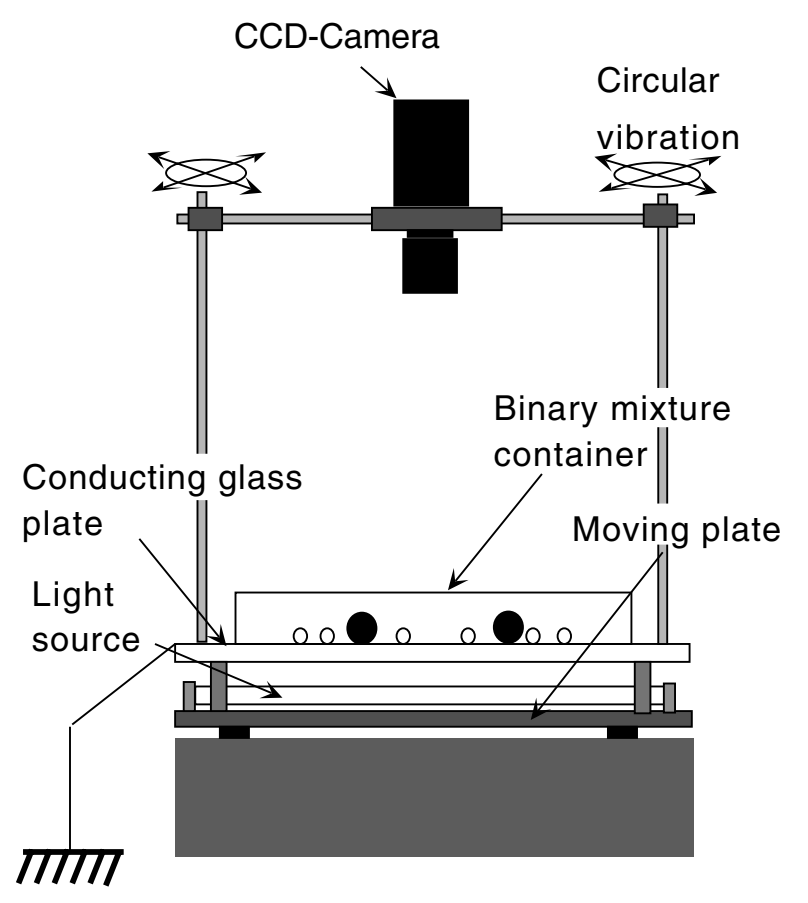

FIG. 1. Sketchy representation of the experimental device. The diameter of the container is $29 \mathrm{~cm}$. The bottom glass plate is coated with an electrically conducting indium-tin oxide layer to suppress static electricity. 


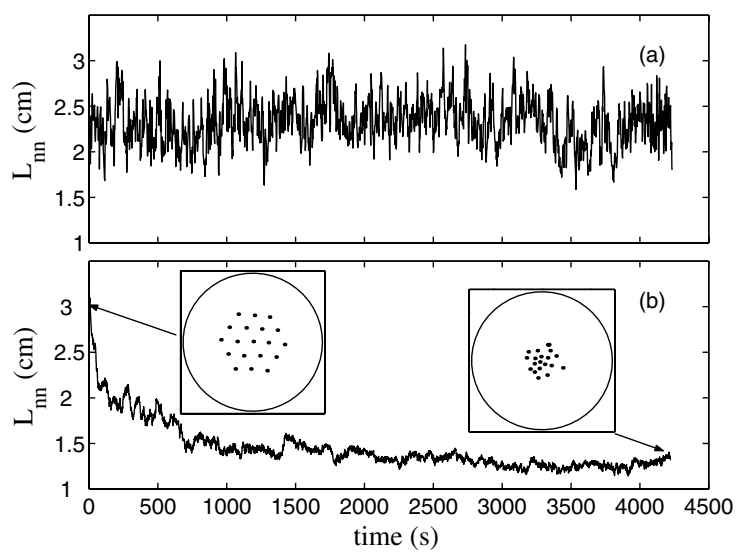

FIG. 2. Temporal evolution of the mean distance, $L_{\mathrm{nn}}$, between nearest neighbors of the 19 large spheres mixed with: (a) 1440 small spheres $\left(\mu_{\mathrm{tot}}=0.30\right)$, and (b) 4320 small spheres $\left(\mu_{\text {tot }}=0.84\right)$. The insets show the initial and final positions of the large spheres. For both curves the driving frequency is $\nu=1 \mathrm{~Hz}$, and the amplitude is $A=1.27 \mathrm{~cm}$.

collapsing process, can be observed. In contrast, at the higher density $\mu_{\text {tot }}=0.84$ a collapse of the large particles occurs within several minutes [Fig. 2(b)]. $L_{\mathrm{nn}}(t)$ decreases from its initially set value $L_{i}=3 D$ and tends to an asymptotic value close to 1 particle diameter $D$. For a clear characterization of the segregation the mean value of the distance between nearest neighbors in the asymptotic regime, $L_{\infty}$, and the standard deviation around it, $\Delta L_{\infty}$, are measured as a function of the filling fraction $\mu_{\text {tot }}$. Both quantities are plotted in Fig. 3 for six different excitation parameters. A sharp decay appears, both in $L_{\infty}$ and $\Delta L_{\infty}$, when a critical value of the filling fraction is

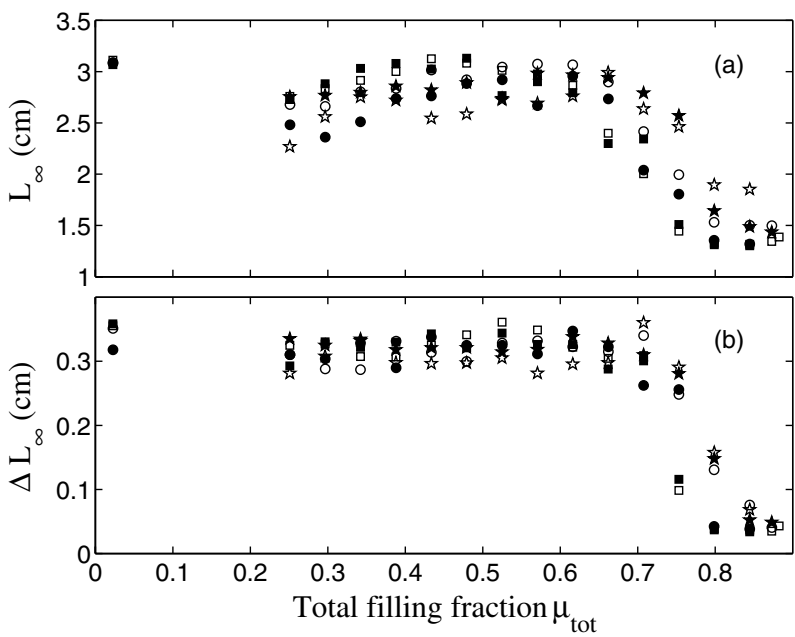

FIG. 3. (a) Dependence of the asymptotic value $L_{\infty}$ of the distance between nearest neighbors on the total filling fraction of particles $\mu_{\text {tot }}$, and (b) the root-mean-square fluctuations $\Delta L_{\infty}$ around this average. For both curves the symbols represent the excitation parameters given in Table I. The points at $\mu_{\text {tot }}=0.02$ show the measurements with the large spheres only. exceeded. Here a transition between a nonsegregated state at low density and a segregated state at high density can be defined. Precise numbers for the critical values of $\mu_{c}^{(a)}$ (and $\mu_{c}^{(b)}$, respectively) are given in Table I. These critical filling fractions seem to be independent of the driving frequency but decrease with increasing shaker amplitude. Only a few data are presented for small filling fractions $\mu_{\text {tot }}<0.5$, because segregation was never observed in this regime.

During the segregation experiments we noticed global changes in the dynamics of the system while crossing the transition threshold. In particular, the smaller spheres became arranged in regular, triangular patches. To study this phenomenon further we investigated the behavior of a layer of monodisperse spheres under the same excitation.

A monolayer of small black glass spheres (diameter $d=0.4 \mathrm{~cm}$ ) is placed inside the dish. After an early stage during which the system loses all traces of the arbitrary initial configuration, an image is taken. To underline the developed structured state a 2D Fourier transformation is performed. Results of these measurements are shown in Fig. 4, for low and high filling fractions of spheres. At low density, the configuration of grains does not show any structures. Its 2D power spectrum displays a continuous intensity distribution within a circle of radius $k_{d}=2 \pi / d$. In contrast, at high density, the small spheres arrange in a triangular lattice. In the $2 \mathrm{D}$ power spectrum six peaks appear, evenly spaced by an angle of $\pi / 3$, at a wave number $k_{0}=2 \pi / \frac{\sqrt{3}}{2} d$. The dynamics of the particles is obviously different in these two regimes. At low density each particle is free to follow its own trajectory until it collides with its neighbor, like in a fluid. On the other hand, at high density, the particles are forced into a collective motion inside a 2D crystal. The crossover between these two regimes is reminiscent of a liquid-solid transition.

To specify the transition point, a characteristic order parameter is extracted from the power spectra. The spectral intensity is integrated radially in an annulus $0.98 k_{0}<$ $k<1.02 k_{0}$ around the expected peaks as shown in Fig. 4. The resulting averaged intensity defines a function of the azimuthal angle $\varphi$. In the structured state this function is supposed to present six equidistant peaks. To obtain a

TABLE I. Values of the critical filling fraction extracted from Fig. 3 for six different excitation parameters. The critical values $\mu_{c}^{(\mathrm{a}, \mathrm{b})}$ are defined as the value of $\mu_{\mathrm{tot}}$, where $L_{\infty}$ (or $\Delta L_{\infty}$, respectively) equals the arithmetic mean of its extrema.

\begin{tabular}{|c|c|c|c|c|c|c|}
\hline Symbol & $\hat{s}$ & $\star$ & $\bigcirc$ & O & $\square$ & $\mathbf{\square}$ \\
\hline$A(\mathrm{~cm})$ & 0.64 & 0.64 & 1.27 & 1.27 & 1.59 & 1.59 \\
\hline$\nu(\mathrm{Hz})$ & 1.0 & 1.5 & 1.0 & 1.5 & 1.0 & 1.5 \\
\hline$\mu_{c}^{(a)} \pm 0.02$ & 0.74 & 0.76 & 0.72 & 0.71 & 0.68 & 0.68 \\
\hline$\mu_{c}^{(b)} \pm 0.02$ & 0.77 & 0.78 & 0.78 & 0.76 & 0.71 & 0.73 \\
\hline
\end{tabular}



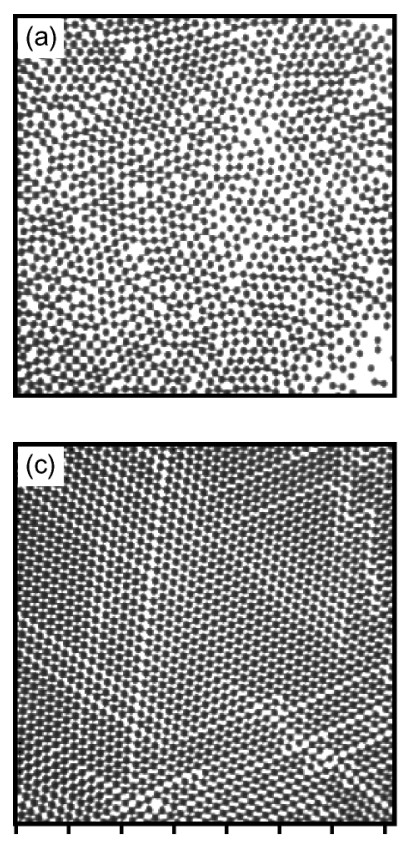

$\begin{array}{llllllll}0 & 5 & 10 & 15 & 20 & 25 & 30 & 35\end{array}$ $x / d$
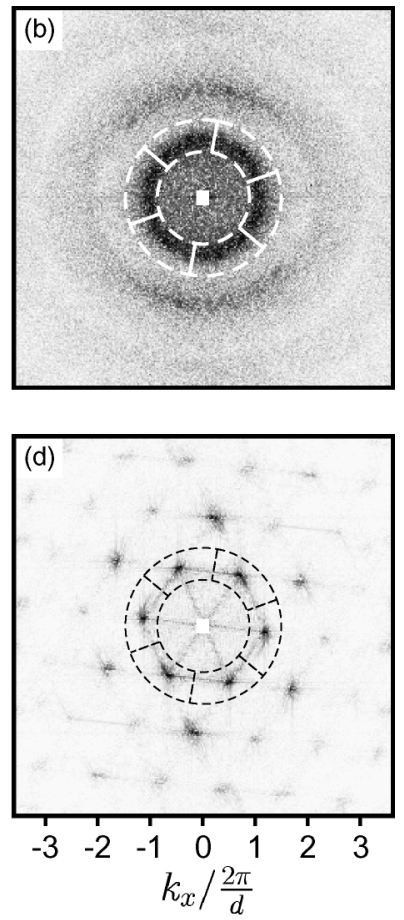

FIG. 4. (a) Image of a monolayer of 2400 small spheres (filling fraction $\mu=0.46$ ) rolling on a table shaken with an amplitude $A=1.91 \mathrm{~cm}$ and a frequency $\nu=1.67 \mathrm{~Hz}$. (b) Plot of the corresponding 2D power spectrum (high intensity appearing black). (c) Image of a monolayer of 4000 small spheres ( $\mu=0.76$ ) under the same excitation, and (d) its 2D power spectrum. The snapshots are taken in a square of size $15 \mathrm{~cm}$ at the center of the cell. The overlays in the $2 \mathrm{D}$ power spectra indicate schematically the regions of $k$ space where the intensity of the spectrum was analyzed quantitatively (see text).

single characteristic number, which quantifies the order of the structured state, the angular space $[0,2 \pi]$ is subdivided into six equal parts. The intensities in each interval are summed up yielding a singly peaked function $I(\varphi)$ in the reduced angular range $\varphi \in[0, \pi / 3]$. Dividing $I(\varphi)$ by its arithmetic mean yields a normalized intensity

$$
I_{\mathrm{n}}\left(\varphi_{i}\right)=\frac{I\left(\varphi_{i}\right)}{\frac{1}{N} \sum_{i=1}^{N} I\left(\varphi_{i}\right)}, \quad \varphi_{i}=i \frac{\pi}{3 N}
$$

from which an order parameter

$$
\alpha \equiv I_{\mathrm{n}, \max }-1
$$

is derived. The open circles in Fig. 5 show that at a low density of spheres, where no coherent structure exists, no peak survives this analytic procedure $\left(I_{n, \max }=1.2\right)$, whereas at a high density a pronounced peak remains (solid circles in Fig. 5). Its height $I_{\mathrm{n}, \max }=5.1$ reflects the degree of symmetry established in the triangular lattice of the particles.

The values of $\alpha$ versus the filling fraction $\mu$ are shown in Fig. 6 for the driving conditions $A=1.91 \mathrm{~cm}$ and $\nu=$ $1.67 \mathrm{~Hz}$. Similar to the segregation experiments, here

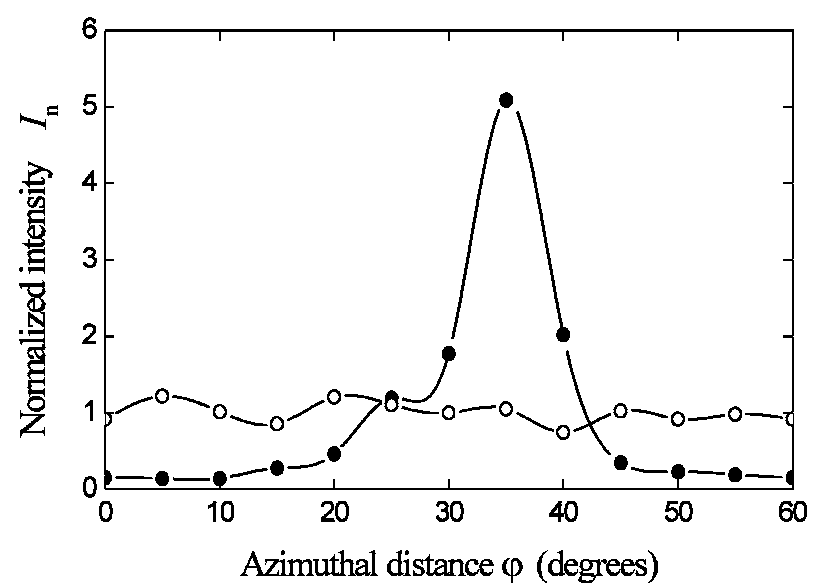

FIG. 5. Normalized intensity $I_{\mathrm{n}}$ of the power spectrum for $\mu=0.46(N=2400, \bigcirc)$, and $\mu=0.76 \quad(N=4000, \bigcirc)$, respectively. Shaker amplitude $A=1.91 \mathrm{~cm}$ and frequency $\nu=1.67 \mathrm{~Hz}$.

again, a transition can be observed around a critical filling fraction.

Since both transitions depend on the shaker amplitude, we explored this dependence further. Figure 7 shows the resulting phase diagram of the critical filling fraction $\mu_{c}$ vs shaker amplitude $A$. The regime where segregation occurs is always slightly above the liquid-solid transition line. This suggests that the granular phase transition is a precondition for segregation.

The transition is in the regime from 0.64 to 0.72 and depends on the amplitude of the driving shaker in a counterintuitive way: The liquid-solid transition line can be crossed by increasing the amplitude and thereby the energy input. A similar phenomenon was coined

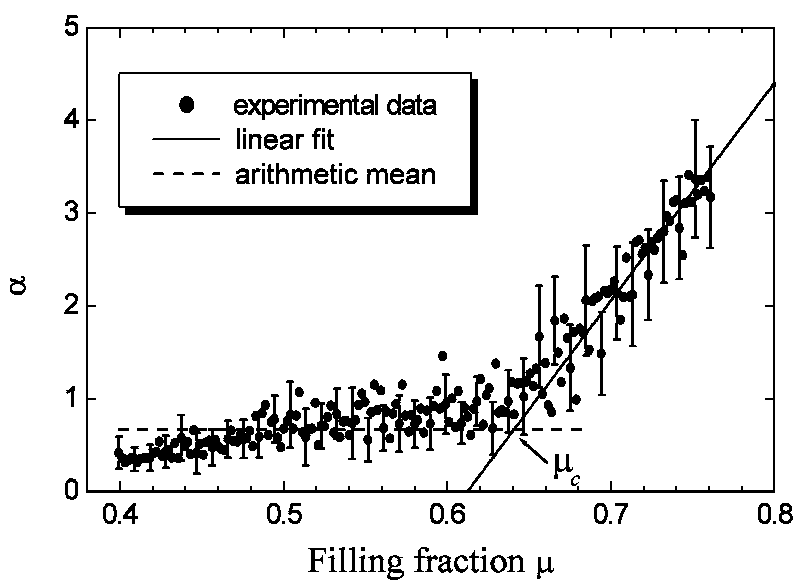

FIG. 6. Evolution of the order parameter $\alpha$ with the filling fraction $\mu$. Each dot represents the analysis of 200 frames with a temporal delay of $16.7 \mathrm{~ms}$. Shaker amplitude $A=1.91 \mathrm{~cm}$ and frequency $\nu=1.67 \mathrm{~Hz}$. The critical point $\mu_{c}=0.64 \pm$ 0.01 is found from the intersection of a linear fit of the data with $\alpha>1.34$ with the mean level of the precritical data. 


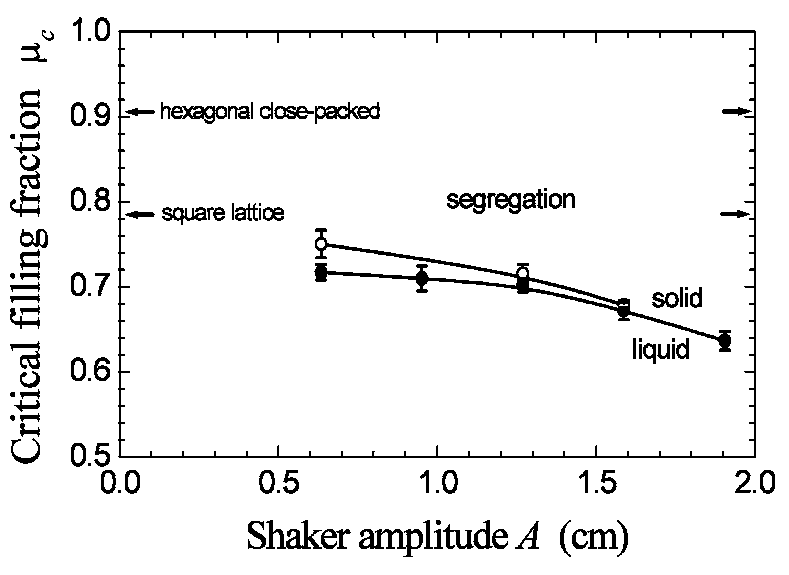

FIG. 7. Phase diagram of the critical filling fraction $\mu_{c}$ vs shaker amplitude $A$ showing the regime $\mu>\mu_{c}^{(a)}(\bigcirc$, data listed in Table I) where segregation occurs. The liquid-solid transition line (O) is obtained from the normalized Fourier intensity data. The arrows point to the filling fractions of a hexagonal close-packed $\left(\mu_{\mathrm{hcp}}=\pi / 2 \sqrt{3}\right)$ and a square lattice $\left(\mu_{\mathrm{sq}}=\frac{\pi}{4}\right)$, respectively.

"freezing by heating" in the context of driven mesoscopic systems [8]. The transition value is well below $\frac{\pi}{4}$, indicated by the arrows in Fig. 7, which is a value one might expect on the basis of the purely geometrical argument connected with the concept of dilatancy [9]. This is explained by the fact that the $y$ axis denotes the global filling fraction, which is the control parameter of the experiment. Because of the spontaneous clustering of particles, the local filling fraction in the condensed patches is larger than the global one.

We offer the following thoughts to elucidate the connection between the segregation in the binary mixture and the phase transition in the monodisperse layer of spheres. It seems that the granular material does not stay uniformly distributed but chooses the configuration which minimizes the energy input for a given number of particles and excitation parameters, in order to reduce the amount of energy to be dissipated. For $\mu \leq \mu_{c}$ all particles move randomly with a uniform distribution; i.e., they prefer not to collide with the lateral wall. Indeed at low densities only very few particle-wall collisions can be observed. We have measured that, in this case, large and small particles roll inside the dish in almost the same fashion. No segregation occurs. On the other hand, if the filling fraction exceeds $\mu_{c}$, a boundary-hitting regime begins. This change can be seen and even heard in the experiment. In this regime the matter is continuously hit by the lateral wall. This process tends to decrease the extension of the monolayer which organizes itself in a more and more condensed state, leading to a triangular lattice, when the excitation is increased even further. Following the ideas of Edwards and Grinev [10], this is also the reason why segregation occurs. Beyond the critical filling fraction the collisions with the lateral wall become the dominant driving mechanism. The granular material is continuously compressed by the collisions with the lateral wall and therefore tends to increase its compactness by organizing itself in a triangular lattice. Since the larger particles disturb this ordering process, the system pushes these intruders into the central region of the dish, thereby reducing the number of holes and defects $[11,12]$. We conclude that the main mechanism for size segregation in our binary system is the compression force exerted by the lateral boundary and mediated by the developing lattice of the smaller spheres.

We currently focus on the dynamical aspects of the collapse, like its dependence on the ratio of the particle's diameters, the number of large particles, or the table acceleration, which should help one to understand this interesting phenomenon better.

Support by DFG through Re588/12 and EU through TMR network "Patterns, Noise and Chaos" FMRXCT96-0085 is gratefully acknowledged.

[1] H. M. Jaeger, S. R. Nagel, and R. P. Behringer, Rev. Mod. Phys. 68, 1259 (1996).

[2] K. M. Hill and J. Kakalios, Phys. Rev. E 49, R3610 (1994).

[3] J. Duran, J. Rajchenbach, and E. Clément, Phys. Rev. Lett. 70, 2431 (1993).

[4] P. M. Reis and T. Mullin, Phys. Rev. Lett. 89, 244301 (2002).

[5] E. Falcon, R. Wunenburger, P. Evesque, S. Fauve, C. Chabot, Y. Garrabos, and D. Beysens, Phys. Rev. Lett. 83, 440 (1999).

[6] G. Strassburger and I. Rehberg, Phys. Rev. E 62, 2517 (2000).

[7] S. Aumaître, C. A. Kruelle, and I. Rehberg, Phys. Rev. E 64, 041305 (2001).

[8] D. Helbing, I. J. Farkas, and T. Vicsek, Phys. Rev. Lett. 84, 1240 (2000).

[9] O. Reynolds, Philos. Mag. 20, 469 (1885).

[10] S. F. Edwards and D.V. Grinev, Phys. Rev. E 58, 4758 (1998).

[11] A. D. Dinsmore, A. G. Yodh, and D. J. Pine, Phys. Rev. E 52, 4045 (1995).

[12] J. Duran and R. Jullien, Phys. Rev. Lett. 80, 3547 (1998). 